Original Article

\title{
Does Surgical Repair Still have a Role for Iatrogenic Tracheobronchial Rupture? Clinical Analysis of a Thoracic Surgeon's Opinion
}

\author{
Sung Kwang Lee, MD, ${ }^{1}$ Do Hyung Kim, MD, ${ }^{1}$ Sang Kwon Lee, MD, ${ }^{1}$ Yeong-Dae Kim, MD, ${ }^{2}$ \\ Jeong Su Cho, MD, ${ }^{2}$ and Hoseok I, $\mathrm{MD}^{2}$
}

\begin{abstract}
Purpose: The choice of surgical repair or conservative treatment for iatrogenic tracheobronchial rupture (ITBR) remains controversial. However, thoracic surgeons consider that surgical repair is an important treatment modality. The purpose of this study was to evaluate the clinical results from the perspective of the surgery-preferred group. Methods: We treated 11 patients (8 women and 3 men; age: $52.6 \pm 22.9$ years) with ITBR from January 2011 to January 2016. A posterolateral thoracotomy or a trans-tracheal approach was performed according to the mechanism of injury.

Results: Nine patients underwent surgery, and all patients received primary repair. Five patients received a right posterolateral thoracotomy, whereas one patient received a left posterolateral thoracotomy. No mortality or morbidity related to the surgery was observed. The mechanical ventilation time was $65.9 \pm 99.2$ hours. The intensive care unit duration was $19.7 \pm 33.3$ days. Two patients received conservative treatment, and all patients died of another disease that was not related to the conservative treatment.

Conclusion: Our mortality or morbidity due to surgery was not higher than world literature results of conservative treatment. We thought surgery is the primary treatment choice for ITBR in the absence of a good indication for conservative treatment.
\end{abstract}

Keywords: iatrogenic disease, trachea, rupture, tracheal surgery

\section{Introduction}

Choosing surgical repair or conservative treatment for iatrogenic tracheobronchial rupture (ITBR) remains controversial. Immediate surgical repair has been the treatment

${ }^{1}$ Department of Thoracic and Cardiovascular Surgery, Research Institute for Convergence of Biomedical Science and Technology, Pusan National University Yangsan Hospital, Yangsan, Gyoungnam, Korea ${ }^{2}$ Department of Thoracic and Cardiovascular Surgery, Pusan National University Hospital, Busan, Korea

Received: August 14, 2016; Accepted: September 5, 2016 Corresponding author: Do Hyung Kim, MD. Department of Thoracic and Cardiovascular Surgery, Pusan National University Yangsan Hospital, Pusan National University College of Medicine, 20, Geumo-Ro, Mulgeum-Eup, Yangsan-Si, Gyeongsangnam-Do 50612, Korea

Email: yumccs@nate.com

(C)2016 The Editorial Committee of Annals of Thoracic and Cardiovascular Surgery. All rights reserved. of choice for tracheal injuries in the past. Recently, conservative treatment has been preferred to primary treatment because mortality and morbidity due to conservative treatment are significantly lower than that following surgery. ${ }^{1-6)}$

However, the question of surgical or conservative treatment for ITBR has not been determined until now. It is difficult to conclude that conservative treatment is superior to surgery despite the fact that the reported absolute mortality due to surgery is higher than that of conservative treatment. The cause of the high surgical mortality was obtained from two reports ${ }^{7,8)}$ because $63.6 \%$ of all mortalities originated from these two reports. Excluding those two reports, the overall mortality rate in surgery-preferred group is $9.2 \% .^{9-19)}$ Moreover, a more detailed analysis of those two reports showed that surgery-related mortality was unexpectedly low $(6.3 \%)$. The study that reported the highest mortality $(61.5 \%)$ did not have repair-site problems, and all causes of death were 


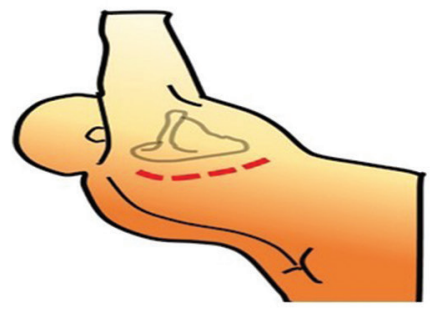

a

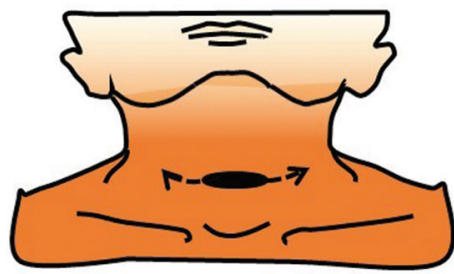

C

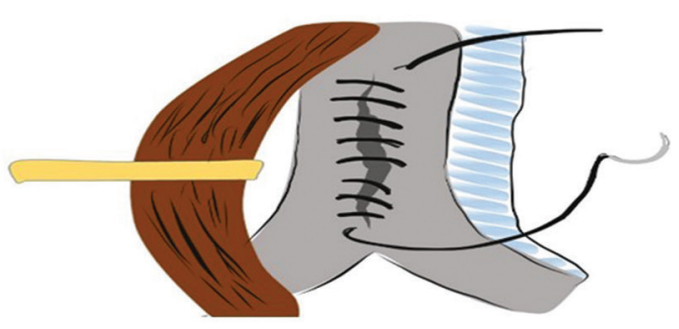

b
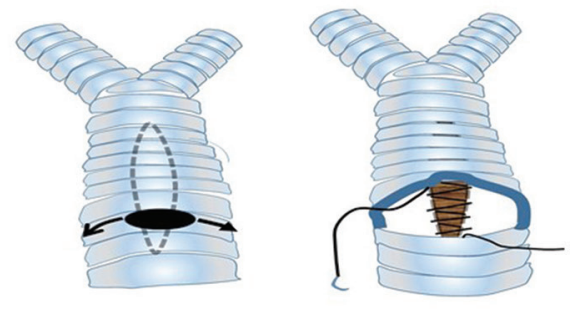

d

Fig. 1 A right lateral thoracotomy was performed through the fifth intercostal space if iatrogenic tracheobronchial rupture developed due to intubation. (a) After removing the mediastinal pleura, the area between the esophagus and posterior trachea was dissected, and the esophagus was retracted using a silicone drain to expose the tear site. Interrupted primary sutures were used to repair the tear (b). If ITBR developed due to a tracheostomy, the tracheostomy skin incision was laterally extended about $1 \mathrm{~cm}(\mathbf{c})$. A full tracheal incision was made lateral to the transverse midline incision in the anterior wall of the trachea. Running absorbable sutures were used to repair the area proximal and distal to the laceration in the posterior wall through the full tracheotomy site (d). ITBR: iatrogenic tracheobronchial rupture

nonsurgical, such as the sequelae from trauma-related hypoxia, underlying diseases (malignancy), or septic multi-organ failure. ${ }^{7)}$ Thus, the prognosis depended on the underlying disease rather than on tracheal damage. ${ }^{20)} \mathrm{Sim}$ ilar results were described in the other study, in which overall mortality from tracheobronchial rupture was $16.8 \%{ }^{8)}$ however, tracheobronchial rupture-related mortality in surgical patients was $2.2 \%$. Therefore, mortality due to tracheal surgery is not higher than that following conservative treatment, if these two reports are excluded.

The purpose of this study was to evaluate the clinical results from the perspective of the surgery-preferred group. We reviewed the literature to confirm the feasibility of surgery for treating ITBR.

\section{Materials and Methods}

We treated 11 patients $(8$ [72\%] women and 3 [27\%] men; mean age: $52.6 \pm 22.9$ years; range: $10-82$ years) with ITBR from January 2011 to January 2016 and retrospectively analyzed the patients' profiles, causes, and extent of tracheobronchial injury; clinical symptoms; chosen treatment option; postoperative ventilation time; and outcome.

Table 1 shows the patients' characteristics. The mean injury size was $4.18 \pm 2.6 \mathrm{~cm}$ (range: $1-10 \mathrm{~cm}$ ) as measured bronchoscopically or directly. The ITBR was located in the cervical trachea in $3(27.2 \%)$ of the 11 patients, the cervicothoracic trachea in six patients (54.5\%), and the thoracic trachea in two patients (18.3\%). One or more of the classic signs and symptoms were observed in all patients, such as subcutaneous emphysema, pneumomediastinum, pneumothorax, hemoptysis, dyspnea, or difficulty with mechanical ventilation. Three cases of ITBR were detected during surgery, and eight were found after intubation. The mean delay in the diagnosis was $64.56 \pm 62.6$ hours (range: $4-312$ hours).

\section{ITBR surgical procedure}

All patients with ITBR that developed due to intubation underwent a right lateral thoracotomy through the fifth intercostal space with one exception, in which a left thoracotomy was performed (Fig. 1a). After removing the mediastinal pleura, the area between the esophagus and 


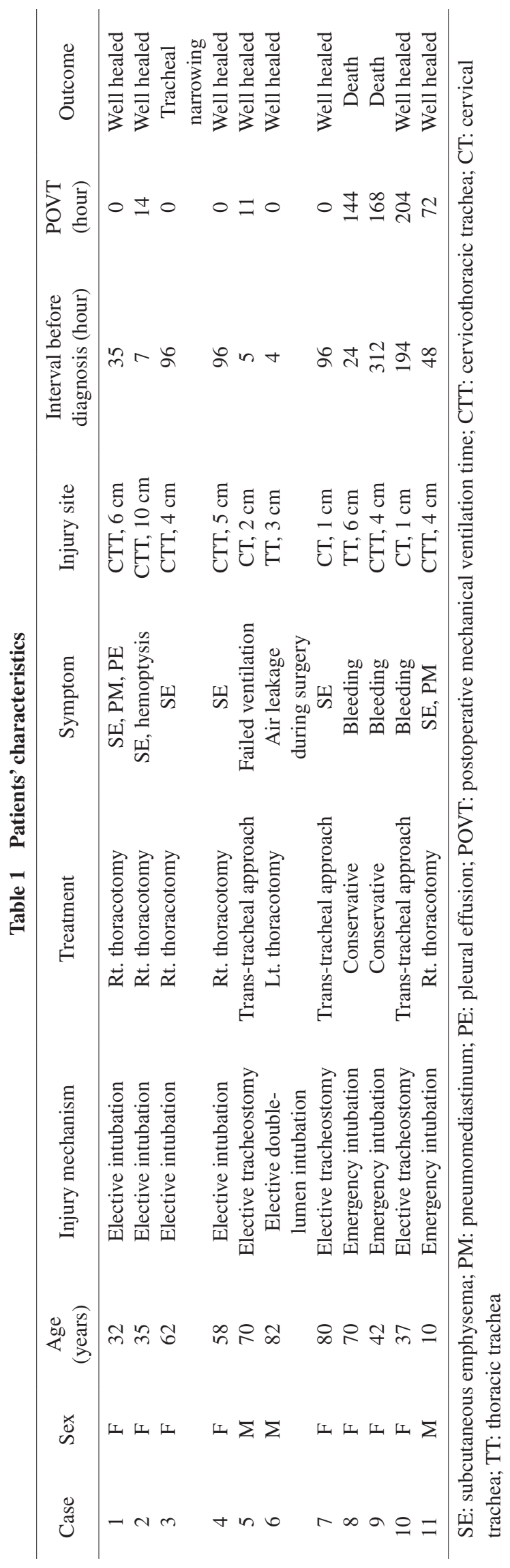

posterior trachea was dissected, and the esophagus was retracted using a silicone drain to expose the tear. Continuous primary sutures were to repair the tear (Fig. 1b).

The tracheostomy skin incision was extended laterally for $\sim 1 \mathrm{~cm}$ in ITBRs that developed due to tracheostomy (Fig. 1c). A cervical neck dissection was performed in the same manner as for cervical trachea surgery. A full tracheal incision was made lateral to the transverse midline incision in the anterior wall of the trachea. Running absorbable sutures were used to repair the area proximal and distal to the laceration on the posterior wall through the site of the full tracheotomy (Fig. 1d). The anterior trachea was closed with everting interrupted sutures, and a central hole remained for the tracheostomy.

\section{Results}

Nine patients underwent primary surgical repair. A rightsided posterolateral thoracotomy in the fifth intercostal space was performed in five patients. The destroyed tracheobronchial wall was reconstructed with interrupted or running absorbable sutures. A tear in the left main bronchus was discovered after an elective double-lumen intubation tube was inserted for video-assisted thoracic surgery during a left upper lobe lobectomy, so conversion to left lateral thoracotomy was necessary to repair the rupture site. Three patients had lacerations in the upper and middle thirds of the trachea by tracheostomy, so a trans-tracheal approach was used for the repair (all three patients had a collar incision due to the tracheostomy). No mortality or morbidity related to surgery was observed. The mean mechanical ventilation time was $65.9 \pm 99.2$ hours (range: 0-280 hours). The duration of stay in the intensive care unit was $19.7 \pm 33.3$ days (range: $1-90$ days). The mean follow-up duration was $1.9 \pm 1.6$ months (range: 0-5 months). One case of tracheal narrowing due to tracheal rupture-related sequela was experienced (Table 2).

Two patients underwent conservative treatment; one of these patients underwent conventional conservative treatment. The latter patient underwent emergent intubation and colectomy due to necrotizing colitis, and the tracheal rupture was confirmed incidentally during the tracheostomy by bronchoscopy. Conservative treatment was used because no rupture-related symptoms or mediastinitis occurred. However, the patient died of postoperative sepsis from the necrotizing colitis. The cause of death was not related to the conservative treatment for ITBR. The other patient underwent extracorporeal membrane oxygenation (ECMO) for sudden massive hemoptysis and 
Table 2 Patient results

\begin{tabular}{lcrrr}
\hline & All & Surgery & \multicolumn{1}{c}{$\begin{array}{c}\text { Conservative } \\
\text { treatment }\end{array}$} & p value \\
\hline Age (years) & $52.6 \pm 22.9$ & $51.8 \pm 24.6$ & $56.0 \pm 19.8$ & 0.431 \\
Time between onset and surgery (hours) & & $64.6 \pm 62.6$ & & \\
Ventilation time (hours) & $65.9 \pm 99.2$ & $33.44 \pm 68.1$ & $212.0 \pm 96.2$ & 0.549 \\
ICU stay (days) & $19.7 \pm 33.3$ & $33.4 \pm 68.1$ & $212.0 \pm 96.2$ & 0.246 \\
\hline ICU: intensive care unit & & & &
\end{tabular}

was diagnosed with a tracheal rupture while changing the tracheostomy tube. The patient required emergency surgery due to a large tracheal rupture near the carina, but could not undergo the surgery because of severe acute respiratory distress syndrome caused by massive hemoptysis. ECMO was maintained in self-respiration status for 28 days until the patient's lung condition improved and the damaged area had healed. She was transferred to the general ward on the day 35 after the tracheal injury. The tracheal rupture resolved completely, but the patient died of other causes 3 months later. The cause of death was not related to the conservative treatment.

\section{Discussion}

In ITBRs, the choice between surgical and conservative treatment may be a difficult one. Most institutes use both the choices depending on the specifics of the case, even at those institutes in which one modality is preferred over the other. In this study, we compared ITBR-related mortality in patients treated by physicians preferring surgical repair with that of patients treated by physicians who preferred conservative approaches. The results are shown in Tables 3 and 4, respectively. The overall mortality in the surgery-preferred group was $16.8 \%$, whereas it was $11.8 \%$ in the conservative treatment-preferred group. The procedure-related mortality rates were $2.2 \%$ and $2.0 \%$, respectively, suggesting that procedure-related mortality did not differ although the absolute mortality rate of the surgery-preferred group was higher than that of the conservative treatment-preferred group. We can conclude that the mortality rate in the surgery-preferred group was not higher than that of the conservative treatment-preferred group except for patients with a good indication for conservative treatment. Therefore, surgery remains an important treatment modality.

Recognition of the appropriate treatment is essential to achieve successful repair. Generally, the indications for conservative treatment are as follows: 1) stable vital signs, 2) spontaneous ventilation, 3) absence of esophageal injury, 4) non-progressive pneumomediastinum with/without subcutaneous emphysema, and 5) no signs of sepsis. ${ }^{1-6)}$

Surgical repair is highly recommended under some clinical situations. First, a tracheal injury of $>2 \mathrm{~cm}$ should be treated surgically. Miñambres et al. ${ }^{20)}$ reviewed the literature and recommended that injuries of $<2 \mathrm{~cm}$ can be treated conservatively.

Second, if the rupture is detected during thoracic surgery, immediate surgical repair is recommended. Ceylan et al. ${ }^{19)}$ reported 18 cases of intraoperative tracheobronchial rupture after double-lumen tube intubation, and all patients were repaired successfully without complications. The authors postulated that immediate surgical repair must be performed if a tracheal rupture develops intraoperatively.

Third, surgery is also highly recommended for ruptures that develop during emergency intubation for mechanical ventilation. Positive-pressure ventilation interferes with the healing of the tear site and induces lethal symptoms, such as severe pneumomediastinum, caused by air leakage. Conti et al. ${ }^{4)}$ reported no mortality from conservative treatment in a spontaneous breathing group but $30 \%$ mortality in a respiratory care group. When continual ventilation is required to treat an underlying respiratory problem, surgical repair should be considered if the patient's condition permits. Additionally, if a patient must be on a ventilator, the rupture site must be considered. Conti et al. ${ }^{4)}$ postulated that conservative treatment is technically feasible for near-carinal and main bronchial lesions in most patients undergoing mechanical ventilation. However, it is very difficult to maintain the airway without mechanical ventilation-related complications in a patient with a near-carinal or main bronchial lesion injury. It is difficult to place the cuff below the ruptured area and keep both lungs ventilated. Although selective lung ventilation is possible, it is difficult to maintain breathing for weeks without pneumonia or mediastinitis until the trachea heals spontaneously.

Finally, surgery must be performed in cases of full-layer injury of the posterior membranous portion of the trachea. Cardillo et al. $\left.{ }^{6}\right)$ reported that lesions are classified into four 

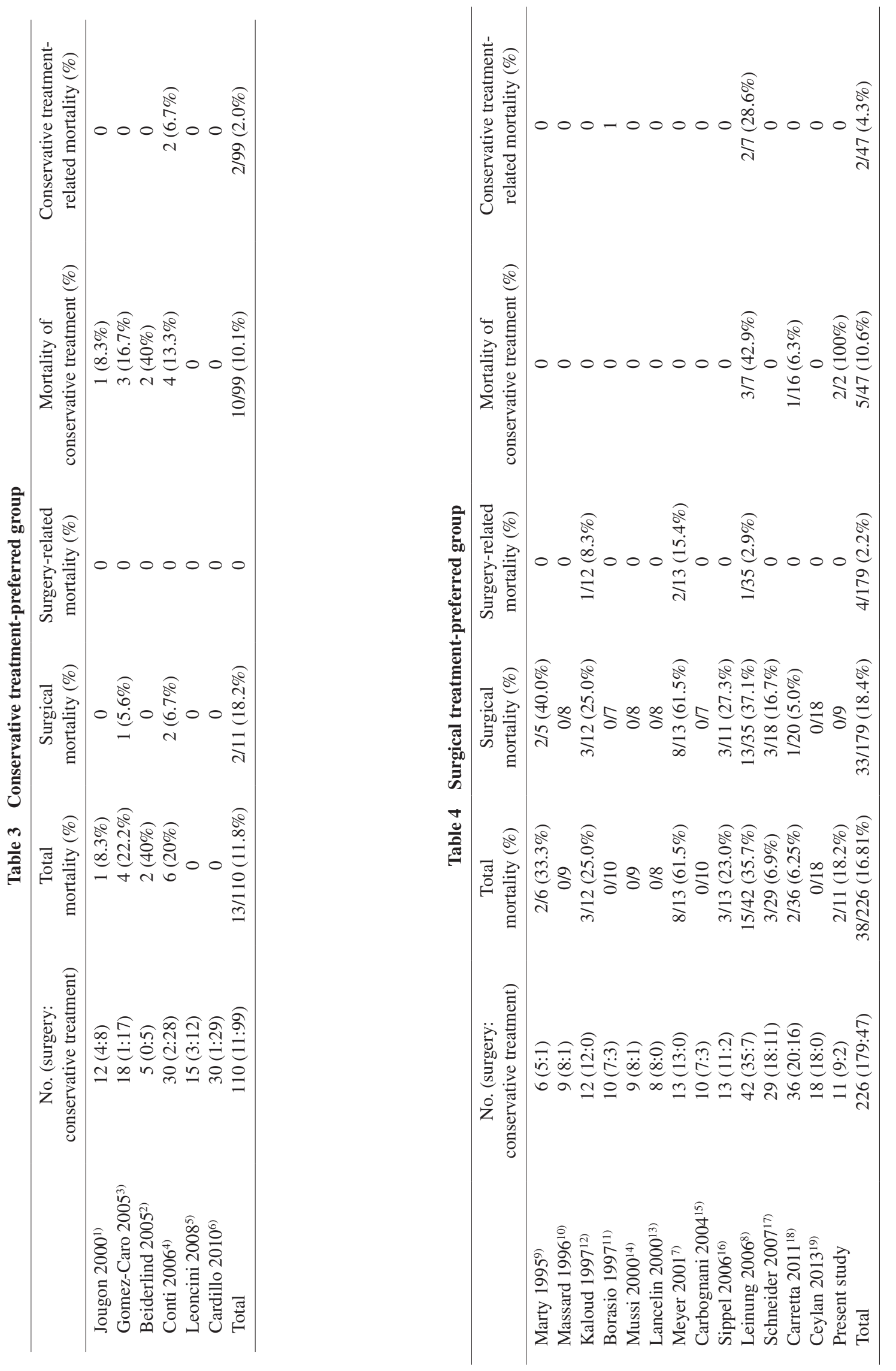
types depending on the depth of the tracheal injury: Level I includes injuries with mucosal or submucosal tracheal involvement without mediastinal emphysema and without esophageal injury; level II includes tracheal lesions up to the muscular wall with subcutaneous or mediastinal emphysema but without esophageal injury or mediastinitis; level IIIA is a complete laceration of the tracheal wall with an esophageal or mediastinal soft-tissue hernia but without esophageal injury or mediastinitis; and level IIIB is any laceration of the tracheal wall with an esophageal injury or mediastinitis. $\left.{ }^{6}\right)$ The authors reported no mortalities and postulated that conservative treatment was possible even with a complete laceration of the tracheal wall if no esophageal injury or mediastinitis was present (level IIIA). We disagree with his opinion. Cardillo et al. ${ }^{6}$ reported that only 2 of 29 conservatively treated patients had level IIIA injuries, which is too few to fully show that level IIIA is a good indication for conservative treatment. We agree that conservative treatment is better than surgery for a partial layer laceration of the posterior membranous portion of the trachea in a patient who is spontaneously breathing. However, prompt surgical repair is better than conservative treatment for a full-layer laceration in a patient undergoing mechanical ventilation.

\section{Conclusion}

In ITBRs, mortality and morbidity are not higher in surgical than in conservative treatment if the severity of the rupture is considered. Many institutes determine treatment modality according to size criteria, vital signs, spontaneous breathing, adjacent organ injury, subcutaneous emphysema, and inflammatory signs. However, in our opinion, in the absence of good indications for conservative treatment, surgery is still the primary treatment choice for ITBR.

\section{Disclosure Statement}

The corresponding author received a research grant from Research Institute for Convergence of Biomedical Science and Technology, Pusan National University Yangsan Hospital, 20, Geumo-ro, Yangsan, Gyoungnam, South Korea; the authors have no conflict of interest.

\section{References}

1) Jougon J, Ballester M, Choukroun E, et al. Conservative treatment for postintubation tracheobronchial rupture. Ann Thorac Surg 2000; 69: 216-20.
2) Beiderlinden M, Adamzik M, Peters J. Conservative treatment of tracheal injuries. Anesth Analg 2005; 100: 210-4.

3) Gomez-Caro Andres A, Moradiellos Diez FJ, Ausin Herrero P, et al. Successful conservative management in iatrogenic tracheobronchial injury. Ann Thorac Surg 2005; 79: 1872-8.

4) Conti M, Pougeoise M, Wurtz A, et al. Management of postintubation tracheobronchial ruptures. Chest 2006; 130: 412-8.

5) Leoncinii G, Iurilli L, Boni L, et al. Treatment of iatrogenic and traumatic tracheal disruptions. Monaldi Arch Chest Dis 2008; 69: 119-27.

6) Cardillo G, Carbone L, Carleo F, et al. Tracheal lacerations after endotracheal intubation: a proposed morphological classification to guide non-surgical treatment. Eur J Cardiothorac Surg 2010; 37: 581-7.

7) Meyer M. Latrogenic tracheobronchial lesions - a report on 13 cases. Thorac Cardiovasc Surg 2001; 49: 115-9.

8) Leinung S, Mobius C, Hofmann HS, et al. Iatrogenic tracheobronchial ruptures - treatment and outcomes. Interact Cardiovasc Thorac Surg 2006; 5: 303-6.

9) Marty-Ane CH, Picard E, Jonquet O, et al. Membranous tracheal rupture after endotracheal intubation. Ann Thorac Surg 1995; 60: 1367-71.

10) Massard G, Rouge C, Dabbagh A, et al. Tracheobronchial lacerations after intubation and tracheostomy. Ann Thorac Surg 1996; 61: 1483-7.

11) Borasio P, Ardissone F, Chiampo G. Post-intubation tracheal rupture. A report on ten cases. Eur J Cardiothorac Surg 1997; 12: 98-100.

12) Kaloud H, Smolle-Juettner FM, Prause G, et al. Iatrogenic ruptures of the tracheobronchial tree. Chest 1997; 112: 774-8.

13) Lancelin C, Chapelier AR, Fadel E, et al. Transcervicaltranstracheal endoluminal repair of membranous tracheal disruptions. Ann Thorac Surg 2000; 70: 984-6.

14) Mussi A, Ambrogi MC, Menconi G, et al. Surgical approaches to membranous tracheal wall lacerations. J Thorac Cardiovasc Surg 2000; 120: 115-8.

15) Carbognani P, Bobbio A, Cattelani L, et al. Management of postintubation membranous tracheal rupture. Ann Thorac Surg 2004; 77: 406-9.

16) Sippel M, Putensen C, Hirner A, et al. Tracheal rupture after endotracheal intubation: experience with management in 13 cases. Thorac Cardiovasc Surg 2006; 54: 51-6.

17) Schneider T, Storz K, Dienemann H, et al. Management of iatrogenic tracheobronchial injuries: a retrospective analysis of 29 cases. Ann Thorac Surg 2007; 83: 1960-4.

18) Carretta A, Melloni G, Bandiera A, et al. Conservative and surgical treatment of acute posttraumatic tracheobronchial injuries. World J Surg 2011; 35: 2568-74.

19) Ceylan KC, Kaya SO, Samancilar O, et al. Intraoperative management of tracheobronchial rupture after doublelumen tube intubation. Surg Today 2013; 43: 757-62.

20) Minambres E, Buron J, Ballesteros MA, et al. Tracheal rupture after endotracheal intubation: a literature systematic review. Eur J Cardiothorac Surg 2009; 35: 1056-62. 